\title{
Kernos
}

Revue internationale et pluridisciplinaire de religion grecque antique

13 | 2000

Varia

\section{Mythes grecs de sacrifice humain en Étrurie Problèmes iconographiques et socio-historiques}

\section{Pierre Bonnechere}

\section{(2) OpenEdition \\ Journals}

Édition électronique

URL : http://journals.openedition.org/kernos/1303

DOI : 10.4000/kernos.1303

ISSN : 2034-7871

\section{Éditeur}

Centre international d'étude de la religion grecque antique

\section{Édition imprimée}

Date de publication : 1 janvier 2000

ISSN : 0776-3824

\section{Référence électronique}

Pierre Bonnechere, «Mythes grecs de sacrifice humain en Étrurie Problèmes iconographiques et socio-historiques », Kernos [En ligne], 13 | 2000, mis en ligne le 21 avril 2011, consulté le 24 septembre 2020. URL : http://journals.openedition.org/kernos/1303 ; DOI : https://doi.org/10.4000/kernos.1303 
Kernos, 13 (2000), p. 253-329.

\title{
Revue des Livres
}

\section{Comptes rendus critiques}

\author{
Mythes grecs de sacrifice bumain en Etrurie \\ Problèmes iconographiques et socio-bistoriques ${ }^{1}$
}

Pierre Bonnechere (Université de Montréal)

Les études d'iconologie ont connu ces dernières années un remarquable renouveau, y compris pour les œuvres habituellement délaissées car artistiquement moins agréables à l'œil. Encore très récemment, les urnes étrusques posaient, pour les chercheurs qui n'avaient pas la chance d'évoluer en Italie, un réel problème d'accessibilité, et il fallait recourir au vieux répertoire de $\mathrm{H}$. Brunn et $G$. Körte pour s'en faire une vague idée ${ }^{2}$, et ce malgré plusieurs corpus en cours de réalisation ${ }^{3}$. Tout le monde connaissait les fameuses séries pérugiennes d'urnes décorées du sacrifice d'Iphigénie, mais là aussi s'arrêtaient les conclusions que l'on pouvait en tirer, à moins de se mettre en chasse dans les publications spécialisées relatives à l'art et l'histoire étrusques. Rien qu'à cet égard, le travail de Steuernagel est méritoire: en appendice, il livre un catalogue des œuvres figurant une mise à mort à l'autel, - sacrificielle ou non - qui comprend quelque 305 entrées où prennent place principalement des urnes, avec de rares peintures murales et quelques sarcophages ${ }^{4}$. Chaque pièce est accompagnée d'une brève description (au besoin sur l'authenticité des pièces), des lieux de découverte et de conservation (y compris les anciennes localisations, ce qui est capital pour les non-spécialistes), une datation au fait des découvertes actuelles et

1 Dirk Steuernagel, Menschenopfer und Mord am Altar. Griechiscbe Mythen in etruskiscben Gräbern, Wiesbaden, Dr. Ludwig Reichert Verlag, 1998, 222 p., 50 pl. horstexte (Palilia, 3). ISBN 3-89500-051-5.

2 H. BRUNN \& G. KöRTE, I rilievi delle ume etruscbe, vol. 1-3, Rome, 1870-1916.

3 Urne volterrane, vol, 1-2, Florence, 1975-1986 (Corpus delle urne etrusche di età ellenistica); G. DAREGGi, Urne del territorio perugino, Rome, 1972 (Quaderni dell'istituto archeologico dell'università di Perugia, 1); M. SANNIBALE, Museo gregoriane etrusco. Le urne cinerarie di età ellenistica, Rome, 1994, etc. Ajoutons-y : Le ciste prenestine, vol. 1 (12), Rome, 1979-1990.

4 Bien que l'on comprenne la logique du choix, on regrettera simplement que certains monuments figurés cormme les vases, les miroirs et les gemmes, dont il est question dans le premier chapitre, n'aient pas été ajoutés au catalogue, d'autant plus que ces objets proviennent, le plus souvent, des nécropoles. Cela oblige le lecteur à feuilleter la première partie de l'ouvrage dans l'attente de tomber sur des cuvres qu'il connaît et qu'il aurait été parfois nécessaire d'interroger plus en profondeur lors de la démarche interprétative. 
enfin la bibliographie complète, au demeurant parfaitement assimilée. Une cinquantaine de planches figurent enfin quelque 150 monuments en bonnes photographies noir et blanc.

Bien entendu, l'intérêt du travail dépasse, et de loin, ceux de la présentation et du catalogage. Ce livre (en fait le remaniement d'une dissertation doctorale plus ambitieuse présentée à l'Université de Hambourg en 19951) est très méthodiquement bâti. Dans un très long premier chapitre, l'A. classe les figurations par mythes, et ils sont nombreux : Troyens au bûcher de Patrocle, Iphigénie, Oreste et Pylade en Tauride, Polyxène, femmes poursuivies [Cassandre, Hélène, Danaïdes], Oreste et Clytemnestre, Télèphe et Oreste, reconnaissance de Pâris, mort de Myrtilos, Oreste et les Érinyes, mort de Tröllos, combats mythiques à l'autel [Étéocle et Polynice], Galates et Gaulois, combats mythiques im Heiligtum [amazonomachie, gigantomachie, centauromachie], sans oublier quelques scènes difficiles d'interprétation. Chaque figuration mythique est ainsi analysée, au besoin par série, et replacée dans son contexte iconographique.

Fort de l'échantillon des 305 pièces du catalogue, l'A. est en mesure d'avancer des chiffres statistiquement acceptables, et son chapitre deux propose une répartition des thèmes mythiques selon la géographie et la chronologie. Grâce aux contextes de découverte, quand on les connaît, l'A. s'efforce dans le même temps de reconstituer, si possible, les horizons sociaux des défunts. Un utile tableau (p. 106) permet de déterminer les trouvailles par région : l'Étrurie du sud et Orvieto n'ont livré de matériel qu'avant 275 , en fait entre 350-250 av. J.-C.; il ne s'agit pas d'urnes, mais uniquement de peintures, fresques et sarcophages, représentatifs d'une aristocratie dominante que l'on retrouve aux commandes des cités. Aucune région ne livre quoi que ce soit - deux occurrences sont trop isolées pour en tirer des conclusions - entre 275 et 225 av. J.-C.; puis l'Étrurie du nord, soient Volterra, Chiusi et Perugia, développe abondamment ces thèmes entre 225 et 75 av. J.-C., surtout des urnes avec cette caractéristique d'une double catégorie d'acquéreurs, non seulement l'aristocratie, mais aussi la «classe moyenne " (terme qui demeure cependant très anachronique). Ce dédoublement de la clientèle devra donner lieu à une double enquête historico-sociale sur la signification des images acquises.

L'A. s'est également donné pour but ${ }^{2}$ de dépasser les approches traditionnelles de l'art étrusque, qui consistent, dans la plupart des cas, à jauger le degré de fidélité de l'œuvre par rapport au mythe grec emprunté, approche qui conclut souvent à l'incompréhension des modèles ou à la banalisation, désormais privée de sens, de motifs à l'origine signifiants. Or le chapitre II a le mérite de mettre en relief que les œuvres «fidèlès » et "infidèles " aux modèles grecs se côtoient, sans qu'il y ait évolution chronologique dans le sens d'une perte progressive de la signification originelle. C'est sur la base de ces prémisses engageantes que le chapitre trois ouvre sans doute à des perspectives des plus originales, en tentant de répondre à cette question: quels sont en Étrurie les modes d'accès aux mythes

1 Une autre partie de la thèse était consacrée à la signification des peintures de la tombe François, aux éventuelles relations entre les figurations mythiques de sacrifices humains dans l'art funéraire étrusque et l'origine des combats de gladiateurs. Une des publications annoncées est parue (Rites funèbres? Etruskische Bilder mytbischer Zweikämpfe und der Ursprung der munera gladiatorum, in Hepbaistos, 15 [1997] p. 69-92), remarquable elle aussi d'un point de vue méthodologique et enterrant définitivement un des mirages étrusques les plus tenaces. L'auteur est un familier de ces sujets depuis la fin des années 1980.

2 Son programme est exposé essentiellement aux p. 11-18, avec un remarquable état de la question sur l'iconographie étrusque. 
grecs, et surtout quelle(s) fonction(s) acquièrent-ils, une fois appréhendés dans la mentalité locale? Parmi les divers groupes isolés dans le chapitre premier, l'A. analyse les passerelles qui conduisirent les artisans à de substantielles contamina tions. L'enquête révèle, de façon un peu attendue, que le sujet mythologique était souvent dominé par des contraintes iconographiques, disons pour faire bref des schémas de figurations, lesquels portent une identité régionale souvent reconnaissable et datable dans ses évolutions.

Mais on s'attend moins à ce qui suit : il apparaît que les Étrusques, qui dénudent Clytemnestre au moment où Oreste va la frapper à l'autel, exactement comme ils figurent Cassandre agrippée au Palladium et menacée de viol par Ajax, se préoccuperaient peu de l'essence mythique des personnages, au contraire des faits, en l'occurrence la personne qui cherche refuge dans un sanctuaire. Et l'A. exemplifie sa pensée en schématisant un type de figuration, précisément celui du suppliant, pour mettre en tableau les composantes systématiques du thème du íétnऽ avec un genou sur l'autel (p. 128-136). L'artisan en fait organiserait toute la scène non en fonction du mythe personnalisé qu'il emprunte, mais bien par rapport à l'idée qu'il entend faire passer : de la sorte, par cette hypothèse d'une contamination radicale ou plutôt même d' « effacement » ou d' " abstraction » des motifs originaux au seul profit des thèmes figurés, Dirk Steuernagel tente de sortir l'iconographie étrusque de cette ornière que sont l'incohérence et les déformations inacceptables dont on lui fait si souvent grief. Sans doute y a-t-il place pour la critique, mais il faut reconnaître que l'idée est excellente et qu'elle fera date dans sa systématisation.

Toute la lumière n'est pas faite, cependant, sur l'interprétation de l'iconographie des œuvres d'art : si l'imagier étrusque vise la représentation d'un concept et non celui d'une légende grecque importée, il reste à introduire dans le tableau les nombreuses inscriptions en étrusque qui, rarement il est vrai pour les cas de sacrifices humains, désignent les personnages. Même si l'abstraction du thème apparaît évidente de par les tableaux de concordance iconographique de l'A., le fait que les Étrusques aient pris la peine d'importer les noms montre qu'ils importaient peut-être davantage que des schémas mythologiques, du moins en certains cas. Il me serait difficile de croire, en effet, que ces noms n'aient renvoyé qu'au simple concept que l'on entendait représenter, Oreste signifiant « un suppliant à l'autel » parmi d'autres : à preuve la tombe François, soit au début de l'époque ici incriminée, où les personnages sont identifiés avec une grande précision, alors qu'un flou se manifeste, c'est vrai, dans les œuvres plus tardives ${ }^{1}$.

De ces constatations sur l'interprétation des scènes mythologiques découlent les trois chapitres suivants : la signification eschatologique des thèmes (re)figurés, la relation entre le thème des morts violentes à l'autel et la pratique étrusque des sacrifices humains et enfin, le rôle, au plan social, du recours à l'imagerie du sacrifice humain.

1 Je pense par exemple au transfert d'Achille au profit d'Ajax égorgeant (selon toute vraisemblance) un prisonnier troyen (cratère en calice du Peintre de Turmuca, ca 330-300 av. J.-C.; Paris, Cabinet des Médailles, 920; J.D. Beazley, Etruscan Vase-Painting, Oxford, 1947, no 31, 1). La position de l'A. lui permet d'évacuer aussi une question qui eût pu lui poser problème: les sacrifices dans les mythes grecs importés (qui ne sont pas aussi dépourvus de liens avec l'histoire réelle dans la mentalité ancienne), comment sont-ils interprétés ? En tant qu'œuvre pie ou impie? La littérature grecque qui les colporte ne fait

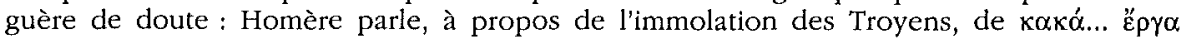
(Iliade, XXIII, 176), et le sacrifice de Polyxène, qui se perpétua surtout dans l'Hécube d'Euripide, n'est à l'avantage ni d'Achille ni des Grecs qui exécutent son désir : autant de questions qui perdent, dans la ligne de pensée ici définie, leur pertinence. 
Selon une théorie bien ancrée, la mort vue comme mors acerba aurait amené les Étrusques à privilégier dans leur choix iconographique les thèmes qui faisaient place à la cruauté, au sang qui coule, à la brutalité qui met fin à la vie. Cette ancienne thèse, au regard de la nouvelle théorie ici développée, est au moins partiellement infirmée par des monuments qui figurent aussi un au-delà heureux, ou du moins une vision eschatologique salvatrice, comme dans les séries d'urnes de Volterra représentant Iphigénie à Aulis soulevée à l'autel mais où la biche de substitution n'est oubliée nulle part.

Cela clarifié, peut-on glaner quelque chose dans une éventuelle relation entre les sources littéraires, lesquelles affirment la pratique de sacrifices humains en Étrurie à l'époque historique, et cette tendance à figurer des sacrifices humains mythiques au Ive siècle et plus encore à l'époque hellénistique ? Question délicate s'il en est eu égard à la bibliographie actuelle qui souvent accepte ce lien. Passant en revue les sources anciennes, l'A. conclut uniquement à la réalité de certains sacrifices de prisonniers, consacrés par devotio à la divinité ${ }^{1}$. C'est la première fois depuis Ambros Pfiffig ${ }^{2}$ que cette question est traitée avec quelque détail, après les nombreuses études des années 1980 sur le sacrifice, animal et humain, dans le monde méditerranéen. Sans doute les conclusions sont-elles, selon moi, encore trop indulgentes pour la plupart des sources, mais tout est une question de point de vue. Voyons les choses plus en détail.

Pour les Phocéens d'Alalia « sacrifiés », en fait lapidés par les habitants de Caere (Hdt., I, 163-167, avec une lacune à l'endroit capital, en I, 167), l'histoire contient tellement d'incohérences qu'il est vraisemblable de conclure à une construction étiologique, au moins partielle, comme le rend manifeste un rapide résumé : les Phocéens, grands voyageurs, reçurent du roi de Tartessos, l'aide financière pour ceindre leur ville d'un haut mur contre la menace perse. Quand Harpage mit le siège devant la ville, les Phocéens, refusant les conditions du Perse, abandonnèrent leur cité sans livrer combat, embarquant femmes, enfants et richesses. Après un détour par Chios, ils reviennent massacrer la garde perse de Phocée, puis tous jurent d'abandonner la cité au prix de terribles imprécations. La moitié de la population finit cependant par retourner à Phocée (malgré le danger des représailles perses [?]), et l'autre moitié se réfugia dans une récente colonie, la Kyrnos-Alalia de Corse, fondée par erreur à la suite d'un oracle oblique dont ils n'apprendraient que plus tard le sens réel. Là, vivant de concert pendant cinq ans avec les colons, fondant des sanctuaires mais aussi se livrant au brigandage, ils encourent la (juste [?]) colère des Carthaginois et des Étrusques sur lesquels ils remportent une victoire tragique dans la mer sarde : vingt bateaux sur soixante, rescapés mais incapables de combattre encore, firent voile sur Alalia, où l'on embarqua femmes, enfants et avoirs (en dépit du nombre ridicule de nefs encore disponibles [?]), avant de cingler vers Rhégion. Quant aux Phocéens qui avaient survécu au naufrage dans la bataille, ils tombèrent aux mains des ennemis (vaincus mais étrangement maîtres de la mer [?]) qui se les seraient partagés et, dans le cas des Caerétins, les auraient lapidés en dehors de la ville. Aussitôt la vengeance de leurs esprits se serait manifestée aux dépens de Caere : tout animal ou toute personne passant près du lieu de la mise à mort en

1 À moins qu'il ne m'ait échappé, je crois n'avoir vu ici la mention de l'article de H.S. VERSNeL, (Saturnus and the Saturnalia, Leyde, 1993 [Inconsistencies in Greek and Roman Religion, 2], p. 136-227, part. 210-227) qui apporte quelques réflexions intéressantes sur le concept de devotio.

2 Religio etrusca, Graz, 1975, p. 110-112. Voir l'approche très neutre et traditionnelle dans le bon manuel de J.-R. JANNOT, Devins, dieux et démons, Regards sur la religion de l'Étrurie antique, Paris, 1998, p. 56-57. 
serait revenu estropié. Face à cela, les Caerétins auraient dépêché à Delphes [?], qui leur aurait enjoint d'instaurer en l'honneur des Phocéens lapidés des sacrifices ainsi que des concours gymniques et hippiques, toutes choses, dit Hérodote, «qu'aujourd'hui encore ils accomplissent».

Je ne sais s'il y a une trame historique derrière ce récit, mais s'il y en a une, il faudrait encore savoir quels éléments accepter et lesquels refuser : en aucun cas on ne se bornera à balayer le côté merveilleux de l'histoire pour ne conserver que les éléments logiquement acceptables, à moins de croire soi-même, par exemple, à la réalité des fléaux décrits par Hérodote ${ }^{1}$. Ce dernier n'est pas un historien au sens actuel du terme, et si ses volontés littéraires et religieuses ne sont pas encore élucidées à ce jour, on peut affirmer avec force que l'ensemble de son travail est partout sous-tendu par le concept de faute et d'expiation, dans une armature qui dépasse de loin le strict rapport historique à la réalité tel qu'on le définit au sens traditionnel ${ }^{2}:$ le seul élément qui puisse être valablement retenu est la coutume caerétine des sacrifices et des jeux perpétrés en l'honneur de " héros » maléfiques et à se rendre favorables. Que ceux-ci soient des Phocéens à rattacher à l'histoire coloniale de la région est tout sauf certain : il suffit de constater que d'autres récits hérodotéens sont longtemps passés pour véridiques avant que leur caractère étiologique n'ait été mis en évidence ${ }^{3}$.

Les scholies glosant l'Ibis d'Ovide (ad 465) ${ }^{4}$ parlent du vou fait par les Étrusques d'offrir le plus fort des Liparéens à Apollon si celui-ci leur donnait la victoire, promesse qu'ils auraient accomplie après l'avoir emporté. Ces scholies, qui commentent un texte à énigme constante, ne représentent pas davantage une source de première qualité : par exemple elles concluent le vers d'après à l'exécution des pharmakoi d'Abdère (ad 467-468, La Penna), alors que la Diegesis de Callimaque, tout en parlant de lapidation, en offre un cinglant démenti (in Aitia [fr. 90], 2, 30-40). La victime chez Ovide s'appelle Théodotus : tout le monde y reconnait une invention post eventum, mais pourquoi l'anecdote devrait-elle être plus fondée que le nom ${ }^{5}$ ? Les exempla cités par Ovide concernent des épisodes très majoritairement mythiques, qui vont de Sisyphe à Busiris. La comparaison de l'exemplum en question avec les Parallela minora de Plutarque est à ce sujet instructive, dans la mesure où quantité de sacrifices humains y sont présentés comme prémices de batailles dont l'historicité peut être rejetée avec certitude (guerres contre les Thraces ou les Pélasges etc.). Quant à la précision alléguée du

1 J'espère d'ici peu publier un article sur ce passage.

2 Perspectives traitées de façon renouvelée par R. GAGNÉ, dans un mémoire de maîtrise réalisé sous ma direction : Hérodote entre l'bistoire, l'ainos et l'oracle, Université de Montréal, 2000.

3 Par exemple le récit en III, 48, qui n'est en fait qu'une élaboration mythique sur un rituel local intégré sur la toile de fond de l'opposition réelle entre Samos et Corinthe: voir J. Ducat, Un rituel samien, in BCH, 119 (1995) p. 339-368, C. Sourvinou-Inwood, "Myth" and History: On Herodotos 3. 48 and 3. 50-53, in "Reading" Greek Culture. Texts and Images, Rituals and Myths, Oxford, 1991, p. 244-284, ainsi que mes remarques, Le rituel samien décrit par Hérodote, III, 48 et la $\beta \omega \mu \mathrm{o} \lambda \chi_{\chi}(\alpha$ spartiate, in LEC, 66 (1998), p. 3-21.

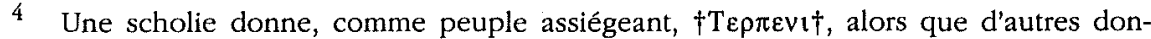
nent Tyrrheni. Une guerre entre les deux peuples est effectivement signalée chez Strabon (VI, 2, 10, C 275) et Pausanias (X, 16, 7).

5 Le fait que Callimaque ait probablement traité de cette histoire dans les Aitia (voir R. Pfeiffer, Callimacbus, 1, Oxford, 1949, ad fr, 93) n'implique pas qu'elle ait été historique : le fragment 91 parle de Mélicertès et les fragments 94-95 d'Hippoménès, un descendant de Codros. 
texte, qui livre notamment le nom du destinataire divin, Apollon, elle n'est certes pas décisive en ce qui concerne l'authenticité du témoignage : citons le vou très précis d'Idoménée qui, menacé de naufrage à son retour de Troie, promet d'immoler à Poséidon le premier homme qui viendrait à sa rencontre ${ }^{1}$ : ce fut son fils. Pausanias, qui citait la guerre entre les deux peuples, se fait l'écho d'un oracle étrange qui aurait permis aux Liparéens de vaincre la flotte tyrrhénienne: de toute évidence cette guerre avait donné lieu à des débordements d'imagination chez les chroniqueurs postérieurs ${ }^{2}$. En fait la scholie prend toutes les apparences d'une explication étiologique traditionnelle de la littérature gréco-latine, qui peut-être remonte à l'époque alexandrine mais qui ne peut servir de preuve en matière de sacrifices humains historiques.

Et ce d'autant moins que le sacrifice des prisonniers de guerre, souvent les plus beaux, est dans l'historiographie ancienne une constante des peuples non grecs (Scythes, Carthaginois, Thraces, Gaulois, Galates, Germains etc.) ${ }^{3}$ : pourquoi les Étrusques, dans le concert des sources grecques et latines, feraient-ils exception quand on les sait associés aux Carthaginois dans leur lutte commerciale et parfois militaire contre les Grecs? De cette constatation découlent aussi mes interrogations sur l'immolation de 307 prisonniers romains par les Tarquiniens en 354, qui nous vient de Tite-Live ${ }^{4}$ : quel crédit lui accorder? Comment être sûr, par exemple, faisant abstraction de la verve livienne sur la Rome outragée qui se défend, que ce massacre à Tarquinia ne constitue pas une explication post eventum pour justifier la mise à mort de 358 Tarquiniens à Rome ? De plus, ce chiffre aux alentours des 300 est récurrent en la matière : Aristomène aurait fait le sacrifice de 300 Spartiates au Zeus du Mont Ithôme ${ }^{5}$, dont le roi Théopompe, - au nom assez proche, faut-il le souligner, du fameux Théodotus des scholies à l'Ibis d'Ovide, - Octave aurait sacrifié 300 sénateurs à Perugia ${ }^{6}$ etc., et il me semble difficile d'entrevoir la vérité entre l'affabulation, la tradition et les explications $a$ posteriori.

Lier les cas entre eux pour tirer des conclusions à plus grande échelle me semble en tous les cas dangereux : dire que les Phocéens furent sacrifiés par lapidation sur la tombe d'une gens de Caere est sans doute totalement abusif (p. 153), comme dire que le sacrifice d'un seul prisonnier à Lipara est l'adoucissement d'une coutume qui exigeait au départ de nombreuses victimes (p. 155). C'est que

1 Servius in Virgile, Énéide, III, 121 et 401; IX, 264; Vaticani mythographi, 1, 195; 2, 210. Autres exemples chez J.G. Frazer (éd.), Apollodomis. The Library, Londres, 1939, p. 394-404 (coll. Loeb). Cf. à ce propos A. Henrichs, Human Sacrifice in Greek Religion. Three Case Studies, in Le sacrifice dans l'Antiquité, Genève, 1981 (Entretiens Hardt, 27), p. 197 : « the known descriptions [de sacrifices humains dans les sources litteraires] have one thing in common : their length and degree of graphic detail stand in reverse proportion to their credibility, historicity and factualness. "

2 Pausanias, $X, 16,7$.

3 Par exemple Démon, 327 F 18a-b Jacoby, Sur cet aspect, voir en dernier lieu J. Rives, Human Sacrifice among Pagans and Cbristians, in JRS, 85 (1995), p. 65-85.

4 Tite-Live (VII, 15, 10) n'est par ailleurs pas corroboré sur ce point par Diodore (XVI, 45, 8). L'A. expose en toute honnêteté l'ensemble des théories sur ce témoignage de Tite-Live, et je dois avouer que celle qu'il défend ne m'apparaît pas la meilleure. Ce qui semble sûr, par contre, c'est l'intention livienne: immolare, quoi qu'on en dise, conserve une nuance proprement religieuse.

5 Clém. Alex., Protr., III, 42. On ignore quelle est sa source, mais Clément cite ce cas dans une liste de sacrifices humains que tous les Grecs devaient connaitre, en tous les cas assez connue pour y jeter les bases de son argumentation apologétique.

6 SuÉtone, Augutuste, 15. 
le raisonnement s'appuie sur l'induction - abusive à mes yeux - d'une règle, le sacrifice de prisonniers, induction réalisée à partir de plusieurs « événements »à l'historicité incertaine. De plus, ces «événements » sont racontés, sur une échelle chronologique qui couvre plus d'un millénaire, par des témoins très différents : Hérodote, dont le projet narratif a ses règles propres, Tite-Live, historien de Rome, avec tout ce que le terme comporte, et les scholies médiévales, aux sources indéterminées, à l'Ibis d'Ovide ${ }^{1}$.

Une divergence de vue de cet ordre est évidemment monnaie courante dans l'interprétation de ce phénomène étrange qu'est le sacrifice humain et, je tiens à le souligner, sans grande importance quant à la solidité du raisonnement qui $s^{\prime}$ ensuit ${ }^{2}$ : l'A. insiste en effet sur le départ à faire entre cette «réalité sacrificielle » et celle que les Étrusques se sont plu à figurer dans leurs monuments funéraires, au demeurant très multiforme, alors en relation possible avec le culte des morts ou des ancêtres : rien d'autre ne les relie donc que le thème, alors que les fouilles sur les sites funéraires n'ont révélé - qui s'en serait étonné ? - que des pratiques sacrificielles animales.

De là l'idée que la figuration de sacrifices humains mythiques, par opposition d'ailleurs aux meurtres impies également figurés sur les mêmes monuments et parfois en vis-à-vis, loin de refléter une pratique réelle ou le souvenir d'une époque lointaine, serait bien plutôt une création " idéologique " contemporaine des urnes, peintures pariétales et sarcophages. Cette "idéologie » aurait consisté en la réinterprétation du passé religieux étrusque, tout à la gloire de l'aristocratie qui, dans ce monde du $\mathrm{IV}^{\mathrm{e}}$ au $\mathrm{I}^{\mathrm{er}}$ siècle, se métamorphosait suite à l'ascension romaine et l'émancipation des classes inférieures. Ce que la nobilitas locale aurait voulu y rappeler, c'est l'époque ancienne où elle avait juste droit de vie et de mort sur les classes soumises (vitae necisque potestas) et, corollairement, la potentielle existence du sacrifice au détriment de ces dernières. La relation entre tenue par les classes élevées entre les thèmes de sacrifice humain et ceux de la violence sacrée mais impie, comme le viol de Cassandre, les meurtres à l'autel (etc.), serait à expliquer donc par une construction aristocratique, consistant à opposer au bon usage de la violence par la noblesse, son utilisation détournée, déviante et chaotique (nous reviendrons sur ce point). Le thème du sacrifice humain serait par là l'attestation réaffirmée d'un pouvoir oligarchique, autant que le fondement de l'autorité religieuse qui devenait la raison d'être des gentes étrusques, dépositaires de l'antique piété envers lẹs dieux et de la connaissance des transgressions à ne pas commettre au risque de retomber dans le chaos.

Ces déductions semblent confirmées par le rôle grandissant joué par la religion et l'érudition religieuse dans l'Étrurie du $\mathrm{rv}^{\mathrm{e}}$ au $\mathrm{r}^{\mathrm{er}}$ siècle avant J.-C., sous l'impulsion de l'aristocratie qui s'en accapare pour préserver un statut privilégié au sein des villes soumises, pour éviter par là l'assimilation par romanisation et encore pour gagner aux yeux des nouveaux maîtres un statut socio-religieux particulièrement important : le culte des ancêtres se voit marqué d'une mythisation et aussi adjoindre le thème des sacrifices humains qui font référence à un lointain passé, lui aussi héröque et réservé aux gentes de souche. Par ailleurs, la reconnaissance romaine envers l'haruspicine locale provoqua en retour une inten-

1 L'enterrement de personnes vivantes, sur le forum boarium notamment, avait fait l'objet d'un excursus (p. 48-50) lors de l'analyse d'un sarcophage (cat., $n^{\circ} 7$ ) sur lequel R. Herbig avait voulu reconnaître, semble-t-il erronément, l'illustration d'un épisode analogue. Les conclusions quant à cette pratique, si elle fut réelle, ne sont pas encore assurées.

2 A l'exception d'une interprétation majeure sur laquelle nous reviendrons. 
sification de la pratique et de l'intérêt religieux auprès des aristocrates étrusques eux-mêmes. C'est donc cette métamorphose socio-religieuse qui, en Étrurie, aurait provoqué l'étonnante efflorescence religieuse qui n'apparaît plus tant comme l'héritière d'une archaïque réalité mais comme l'aboutissement de la réappropriation du passé étrusque par les élites. Je laisse aux spécialistes de la religion étrusque l'appréciation de cette brillante idée qui a le mérite de concevoir la religion comme partie intégrante d'une société qui évolue, plutôt que d'accepter l'image toute faite et intemporelle que nous en ont donnée les sources romaines.

Ces quelques lignes ne peuvent épuiser la pensée de l'auteur, toujours bien étayée. Souvent à contre-courant, parfois iconoclastes, ses interprétations ne risquent pas de passer inaperçues. C'est la première fois que l'on rejette aussi radicalement les visions évolutionnistes du sacrifice humain en Étrurie, dont une des chefs de file demeure Larissa Bonfante ${ }^{1}$, et que l'on axe la recherche sur la dimension symbolique du thème. À défaut d'être l'unique acceptable, cette voie reste néanmoins celle qui a le plus fait progresser le savoir sur ce sujet et qui offre encore le plus de pistes à explorer, sans pour autant d'ailleurs exclure la possibilité d'immolations humaines dans les cultures antiques.

Démarche pleine de sens, l'interprétation du symbolisme que revêt le sacrifice humain demeure, malgré toutes les précautions que l'on peut prendre, grevée de nombreuses incertitudes et plusieurs points, dans les interprétations de l'A., seront évidemment à discuter plus avant. La grande difficulté d'un tel sujet tient fondamentalement aux sources et à la complexité presque insondable de l'approche. Jugeons d'abord des témoignages disponibles: les sources littéraires, seul et unique secours, sont romaines et grecques, et évidemment d'interprétation délicate. Les Étrusques furent les ennemis de Rome, mais aussi leurs très respectés modèles en matière de religion lors de leur déclin, progressivement entourés d'une aura d'étrangeté respectable. Ils comptèrent parmi les bons clients des Grecs mais aussi parmi leurs adversaires commerciaux, et sans aucun doute furent-ils victimes des habituels quolibets sur les étrangers bizarres, modèles d'anti-Grecs. Les arts figurés, quant à eux, bien que très influencés par la tradition grecque, sont évidemment étrusques et sont modelés selon une mentalité qui nous échappe parce que nous ne la devinons qu'à grands traits à travers des sources romaines pour la plupart très tardives. Alors, a priori, que dire de la figuration, dans une tombe étrusque du II $^{\mathrm{e}}$ siècle, d'un mythe comme celui d'Iphigénie, conçu et exporté par des Grecs, mais récupéré par la pensée étrusque ? La question croît encore en difficulté lorsqu'on s'accorde à donner à cet « emprunt » un sens second - déjà si difficile à percer pour la Grèce où les témoignages sont cent fois plus nombreux et explicites - quand la littérature étrusque est perdue à tout jamais.

Dans les interprétations de l'A., il en est une qui laisse le lecteur partagé entre son ingéniosité et sa témérité : l'association réalisée entre la représentation figurée de sacrifices humains avec la religio et la pietas, et celle de la mise à mort sacrilège à l'autel avec le sacrilegium (Chap. 5.3 et 6). Cette hypothèse, indubita blement brillante, appelle quelques commentaires. Dans la religion grecque, mieux connue en raison des sources écrites directes, les sacrifices humains ne

1 L. Bonfante, Historical Art: Etruscan and Early Roman, in AJAH, 3 (1978), p. 147148; EAD., Human Sacrifice on an Etruscan Funerary Urn, in AJA, 88 (1984), p. 531-539; EaD., Un'urna chiusina con "têtes coupées" a New York, in Studi di anticbità in onore di Guglielmo Maetzke, t. 1, Rome, 1984, p. 143-150. 
sont jamais qu'une abomination : par le passé, les hommes furent contraints par les dieux à en accomplir pour sauvegarder les chances de survie de la communauté. Au sens du respect de la volonté divine, on peut l'entendre comme un acte religieux, mais toujours extrême, seule solution pour sortir d'une situation renversée où les règles habituelles sont inopérantes. Privé de sa justification divine, l'acte devient déviant, et la tragédie s'est repue de ce motif. Dirk Steuernagel le reconnaît, mais dissocie sur ce point la mentalité étrusque de la mentalité hellénique (p. 164-165). Il prend ici appui sur ses conclusions à propos de l'historicité «éprouvée » des sactifices de prisonniers chez les Étrusques, que nous avons discutée plus haut et à laquelle je n'avais pu donner mon appui, pour affirmer que ce rite n'était pas ressenti, comme en Grèce, comme une atrocité : des pré misses dépendent évidemment les conclusions. J'aimerais simplement, en jouant à l'avocat du diable, souligner quelques autres pistes de réflexion qui me semblent valoir la peine aussi d'être explorées et dont certaines risquent elles-mêmes, j'en suis conscient, d'être vouées au démenti.

Un bon point de départ pourrait être la série d'une trentaine d'urnes pérugiennes avec le sacrifice d'Iphigénie, toutes ou presque à contenir la fameuse biche de substitution, qui est la manifestation salvatrice de la divinité : la théorie monolithique d'une mors vue comme acerba, sans aucun doute, ici se fissure; mais cette manifestation salvatrice ne s'accorde-t-elle pas assez difficilement, elle aussi, avec le nouveau concept proposé de vitae necisque potestas véhiculé par l'aristocratie locale et tôt récupéré par les classes moyennes? Il faudrait y voir à l'œuvre la puissance aristocratique combinée à la magnanimité de la divinité envers les victimes que celle-ci a elle-même demandées. Mais à ce niveau de conjecture, on finit par perdre le contact avec la réalité historique. D'autres mythes offrent par ailleurs cette caractéristique de la salvatio in extremis : Oreste en Tauride est figuré toujours au moment crucial de la lettre qui va permettre, l'instant d'après, la reconnaissance et l'abandon consécutif du sacrifice ${ }^{1}$.

C'est donc aller un peu vite que d'abstraire ce côté salvateur pour faire primer celui de "sacrifice » au service d'une élite. D'autant que d'autres légendes pourraient être adjointes à celles qui ont été abordées par l'A., notamment celle d'Andromède offerte au monstre sur le vouloir divin et sauvée par Persée. Les figurations de cette légende sont très nombreuses dans la céramique de l'Italie du sud au $\mathrm{IV}^{\mathrm{e}}$ siècle, et on en retrouve un exemple jusque dans la céramique étrusque de la même période ${ }^{2}$. Sans doute y manque-t-il l'autel, puisque l'offrande n'est pas traditionnelle, mais la similitude de sens vaut bien qu'on s'y arrête, et ce surtout quand on connaît cette scène sur des cistes ${ }^{3}$, des reliefs en provenance de temples ${ }^{4}$ ainsi que sur des urnes de Volterra dont la datation ( $\mathrm{ca}$

1 Dans le domaine des mises à mort impies, Télèphe qui menace le petit Oreste à l'autel ne mettra pas son projet à exécution de même que Pâris réfugié à l'autel, Hélène, les Danä̈des et Oreste poursuivis, échapperont à la mort. En d'autres cas, l'immolation ou la mise à mort est menée à terme, comme pour les Troyens au bûcher de Patrocle, Polyxène, Cassandre, Myrtilos, Troïlos, Étéocle et Polynice.

2 Coupe à figures rouges d'un Peintre du Groupe de Sokra, rve siècle; Berlin, 3973; LIMC, art. Andromeda, 30a = art. Perseus, 192 (ill.). Le thème passera à la postérité latine, avec une étonnante vitalité dans les représentations, pour la plupart funéraires, des provinces romaines.

3 Ciste prénestine, $\mathrm{IV}^{\mathrm{e}}-\mathrm{IL}^{\mathrm{e}}$ siècles; Rome, Villa Giulia, 13064; LIMC, art. Andromeda, 145. Ciste prénestine, ca 300-250 av. J.-C.; Paris, Louvre, 1664; LIMC, art. Andromeda, 144 (ill. chez K. Phillips, Perseus and Andromeda, in AJA, 72 [1968], pl. 14, fig. 41).

4 Fragment de relief en terre cuite, Bolsena, ${ }^{\mathrm{e}}{ }^{\mathrm{e}}$ siècle; Florence, Musée archéologique; LIMC, art. Andromeda, 26a (ill. chez A. ANDREN, Arcbitectural Terracotas from Etrusco- 
150-100 av. J.-C.) et l'origine s'intègrent à la perfection dans le tableau chronologique (p. 106) de Steuernagel ${ }^{1}$. La légende d'Hésione, en tous points similaire à celle d'Andromède, est elle aussi présente dans l'imaginaire étrusque des $\mathrm{IV}^{\mathrm{e}}$ et $\mathrm{III}^{\mathrm{e}}$ siècles ${ }^{2}$, mais jamais, jusqu'à présent, sur des urnes. Pour ce type de figurations, une interprétation plus classique pourrait, partiellement du moins, rentrer en ligne de compte (elle s'accommode aussi de l'interprétation de l'A., puisque dans les deux cas on demeure dans l'aristocratie mythique). Pour en revenir en même temps à ce problème d' «imprécision » dans l'emprunt des mythes grecs, on sait combien, dans la peinture de vases grecque d'Italie du sud, les sujets figurés à vocation eschatologique traduisent, bien plus qu'une légende strictement codifiée, une idée, une impression générale qui puise sans trop de précision à la mythologie ${ }^{3}$.

Comme les urnes cinéraires présentent souvent une scène unique, tantôt de " mort à l'autel » ressentie par l'A. comme sacrilège, tantôt de sacrifice humain au contraire manifestation de l'« antique » religio étrusque, il faut supposer un choix iconographique signifiant dont les raisons ne sont pas abordées. De plus, comment pouvons-nous être sûrs des critères selon lesquels les Étrusques auraient classifié les légendes dont ils importaient l'essence? En d'autres termes, pourquoi auraient-ils considéré comme déviant le meurtre de Clytemnestre à l'autel et comme justifiée la mise à mort de Polyxène sur la tombe d'Achille? Selon des critères grecs, étrusques, ou grecs revus par les Étrusques? L'A. en arrive à cette conclusion par la constatation de l'opposition des deux motifs sur de nombreuses œuvres recensées 4 .

Pour tenter d'aller plus loin, attardons-nous à l'analyse proposée par l'A. pour un sarcophage de Tarquinia : l'œuvre présente, sur un long côté, respectivement l'égorgement des prisonniers troyens et le viol de Cassandre, de part et

Italic Temples, Leipzig, 1940, pl. 78, $\mathrm{n}^{\circ}$ 267). Fragment de relief en terre cuite, Civita Castellana (Faléries), ca 250-200 av. J.-C.; Rome, Villa Giulia, 26776; LIMC, art. Andromeda, $146 a$ (ill.).

1 Quatre urnes de Volterra de la deuxième moitié du II $\mathrm{e}^{\mathrm{e}}$ siècle av. J.-C. : Florence, Musée archéologique, 78.486 (albâtre); LIMC, art. Andromeda, 28 (ill.) = art. Kepbeus, 27. Volterra, Musée Guarnacci, 330 (albâtre); LIMC, art. Andromeda, 29 (ill.) = art. Kepheus, 26. Florence, Palais Aldobrandini (jadis Palais Antinori) (albâtre); LIMC, art. Andromeda, 27 (ill.) $=$ art. Kepbeus, 25 (ill.). Volterra, Musée Guarnacci, 331 (tuf); LIMC, art. Andromeda, $30=$ art. Kepbeus, 28 (ill.). Voir peut-être aussi une urne hellénistique de Chiusi; Berlin, 1287; Rilievi, III, p. 242-244, pl. 59-61.

2 Kélébè à figures rouges du Peintre du Groupe de Volterra, ca 350-320 av. J.-C.; Perugia, Musée national; LIMC, art. Hesione, 266 (ill.). Miroirs : Atelier d'Étrurie centrale, ca 325-300 av. J.-C.; coll. Ortiz (Suisse); LIMC, art. Hesione, 268 (ill.). Origine incertaine, ca III $^{\mathrm{e}}$ siècle av. J.-C.; Perugia, Musée national; LIMC, art. Hesione, 267 (ill. dans E. Gehrard, Etruskiscbe Spiegel, 5, pl. 65). Origine incertaine et lieu de conservation inconnu, III ${ }^{\mathrm{e}}$ siècle av. J.-C.; LIMC, art. Hesione, 269 (ill.).

3 La recherche de K. Schauenburg tend à le montrer depuis de nombreuses années, avec toujours plus d'intensité; voir surtout : Zur Grabsymbolik apuliscber Vasen, in JDAI, 104 (1989), p. 19-60. En somme, les problèmes d'« infidélité » aux mythes grecs s'appliquent également, même si de façon moins évidente, aux représentations grecques.

4 Je relève, rapidement, ces associations en opposition : Troyens \& Cassandre (Cat., $\mathrm{n}^{\circ} 1$; 6); Troyens \& Polyxène (Cat., $\mathrm{n}^{\circ} 3$ ); Troyens, Polyxène \& Cassandre (Cat., $\mathrm{n}^{\circ}$ 5); Iphigénie \& Danaïdes, Barbare (?) (Cat., $\mathrm{n}^{\circ} 7$ ); Polyxène \& Télèphe-Oreste, Clytemnestrefuite d'Oreste (Cat,, $n^{\circ}$ 69). Que faire cependant de ceci : Clytemnestre-fuite d'Oreste \& reconnaissance de Paris (Cat,, $\mathrm{n}^{\circ} 72$ ), association sans sacrifice, donc sans opposition? Assigner aux deux figurations le même sens? Dans ce cas, pourquoi ne pas agir de même avec les six autres cas? 
d'autre d'un même autel, et sur un petit côté le sacrifice de Polyxène (?) ${ }^{1}$. L'égorgement des Troyens renverrait à l'《ancienne coutume » des sacrifices humains des aïeux étrusques, coutume authentique mais mise en veilleuse par l'aristocratie, tandis que le viol de Cassandre renverrait à l'acte déviant, toujours lourd de conséquences pour les humains ${ }^{2}$. Sur le côté, la mise à mort d'une femme difficilement identifiable, d'un coup d'épée dans la poitrine, renvoie soit à un sacrifice (Polyxène) et à la coutume ancestrale, soit à une mise à mort déviante renvoyant au viol de Cassandre. Posons cependant la question : pourquoi les deux ne renverraient-ils pas à un acte déviant ou à un acte "ancestral », le premier étant pour ainsi dire posé comme l'équivalent du second? Car au total, si les imagiers étrusques ont abstrait le sens premier des mythes importés, comment être sûr de la signification qu'ils ont conférée à leurs compositions ainsi resémantisées?

Avant de conclure, revenons un moment à la tombe François : près du sacrifice des prisonniers troyens se trouvent des combats mythiques grecs et des combats «historiques » associés à la délivrance d'un prisonnier par Mastarna (le Servius Tullius de la Table Claudienne de Lyon). Le sacrifice est offert par des Grecs dûment nommés : l'artiste, non plus que le concepteur de la décoration, n'a jugé bon de projeter cette immolation dans le monde étrusque du passé. Cette constatation prend davantage de valeur quand on voit à quel point les sacrifices humains figurés par les Étrusques sont, à de rares exceptions près (?), tous grecs. Je pense qu'il serait naif de supposer que les Étrusques, peuple familier de l'immolation animale, ne disposaient pas de thèmes analogues dans leur mythologie, et qu'ils auraient été contraints de les emprunter à leurs voisins, à un moment où les cités grecques d'Italie sont toutes passées sous domination romaine. Comment rendre compte de ces particularités ? Comme souvent, l'hypothèse unique et globalisante ne risque-t-elle pas de dénaturer la réalité, souvent plurielle? Enfin, les monuments funéraires avec représentation de mise à mort à l'autel ne forment qu'une partie de l'iconographie grecque reprise par les imagiers étrusques. Ne court-on pas le risque d'affaiblir son interprétation en centrant l'attention sur une partie de la documentation, à laquelle on peut confier un sens cohérent qui serait plus difficile à maintenir dans le cadre d'une approche globale?

Cette belle thèse de Dirk Steuernagel, avec toutes ses qualités, se distingue des études « épigoniques » qui rabâchent en les précisant des hypothèses devenues certitudes à force d'être inlassablement répétées. Elle était par ailleurs tellement stimulante qu'elle m'a semblé mériter, plutôt que de fades félicitations, quelques passes d'armes que je veux très amicales. Ces dernières ne remettent pas en question l'avancée fondamentale que constitue l'hypothèse d'une conjonction nette entre l'iconographie, à prendre au second degré, et l'évolution politico-sociale de la région.

Dans le domaine du sacrifice humain antique, - comme en tout sans doute mais avec une charge davantage émotionnelle - nous demeurons tributaires de nos idées, voire de nos préjugés : là où l'un conclut à l'historicité des immolations de prisonniers chez les Étrusques, un autre a tendance à conclure au

\footnotetext{
1 Cat., $\mathrm{n}^{\circ} 5, \mathrm{pl}, 4,1$ et 5, 2 .

2 D. Steuernagel précise la même association dans la Tombe François (Cat., $\mathrm{n}^{\circ}$ 1), mais je crois que d'autres scènes aussi y figurent, notamment le combat fatal d'Étéocle et
} Polynice. 
contraire, à partir des mêmes sources et avec les mêmes règles de la critique historique. De là une divergence essentielle : pour Steuernagel, les Étrusques ne sont pas rebutés à l'idée du sacrifice humain; pour moi, ils le sont tout autant que les Grecs et les Romains ${ }^{1}$ et ainsi de suite. Pour pallier ces divergences d'interprétation, nullement irréversibles, une réflexion commune s'impose, incluant évidemment l'historiographie toujours plus pernicieuse qu'on ne le pense, et à laquelle doivent participer des représentants de toutes les disciplines incriminées, historiens et historiens des religions, anthropologues et archéologues. Il y a du pain sur la planche, et tant que ce travail ne sera pas effectué, tout comparatisme au niveau méditerranéen sera voué au plus cuisant des échecs ${ }^{2}$.

\title{
Mitografia di Cassandra: un recente contributo ${ }^{3}$
}

\author{
Sabina Mazzoldi (Università di Urbino)
}

Nell'ultimo decennio la figura di Cassandra è stata argomento di numerose dissertazioni di dottorato, in particolare, ma non solo, nelle università tedesche ${ }^{4}$. Una tale fioritura di studi, giustificata dalla poliedricità del personaggio stesso e dalla sua grande, ininterrotta fortuna nella cultura europea, è stata indubbiamente stimolata dalla pubblicazione dell'importante e fortunato romanzo di Christa Wolf, Kassandra (1983). Molti di questi lavori, infatti, prendono sì le mosse dai testi classici greci e latini, fonti essenziali per la definizione dei caratteri primi del personaggio, ma focalizzano l'indagine sul suo Fortleben nella letteratura medievale, moderna e contemporanea, con approcci non tanto filologico-letterari, quanto di tipo filosofico e/o psicologico, oppure pertinenti alla teoria della letteratura.

Nella sua monografia, anch'essa sviluppo di una tesi di dottorato, la Neblung ritorna, invece, ad una indagine di stampo tradizionale. L'A. procede ad un sistematico esame di tutte le fonti letterarie, greche e latine, relative al personaggio di Cassandra, ordinate cronologicamente: epos e lirica (I), tragedia greca (II), lettera-

\footnotetext{
1 Nous laisserons les pauvres Carthaginois de côté pour le moment.

2 A titre d'exemple, l'essai malheureux de J.E. Miller, The Western Paradigm. Greek and Hebrew Traditions, San Francisco/Londres/Behtesda, 1996.

3 D. Neblung, Die Gestalt der Kassandra in der Antiken Literatur (Diss. Berlin 1996), Stuttgart und Leipzig, 1997.

4 Cf. B. FeICHTINGER, Kassandra und die Dichter. Studien zu Mythographie antiken Autoren, Diss. Salzburg, 1988; T. Epple, Der Aufstieg der Untergangsseberin Kassandra. Zum Wandel ibrer Interpretation vom 18. Jabrbundert bis zur Gegenwart, Diss, Wurzburg, 1992 (pubbl. 1993); S. Müller, Kein Brautfest zwiscben Menscben und Göttern. Kassandra-Mytbologie im Licbte von Sexualität und Wabrbeit, Diss. Dusseldorf, 1993 (pubbl. Köln, 1994); S. Jentgens, Kassandra. Spielarten einer literariscben Figur, Diss. Osnabrück, 1994 (pubbl. Hildesheim/Zürich, 1995); meno recente K. LEDERGERBER, Kassandra. Das Bild der Prophetin in der antiken und insbesondere in der alteren abendländiscben Dicbtung, Diss. Freiburg, 1951; $c f$. anche A. Brault Pascal, Prophetess Doomed. Cassandra and the Representation of Trutb, Diss. NewYork, 1990; S. MazzoLDI,

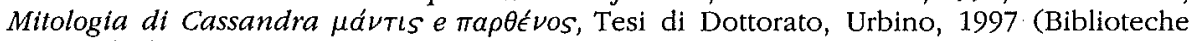
Nazionali di Firenze e Roma).
} 